\title{
Spatially resolved acoustic spectroscopy towards online inspection of additive manufacturing
}

\author{
D Pieris, R Patel, P Dryburgh, M Hirsch, W Li, S D Sharples, R J Smith, A T Clare and M Clark
}

\begin{abstract}
High-integrity engineering applications such as aerospace will not permit the incorporation of components containing any structural defects. The current generation of additive manufacturing (AM) platforms yield components with relatively high levels of defects. The in-line inspection of components built using AM can provide closed-loop feedback and vary build parameters during fabrication to minimise defects. This article reviews the capability of spatially resolved acoustic spectroscopy (SRAS) to be used as an inspection tool for detecting defects and characterising microstructure in parts induced by variations in build parameters. The authors first correlated changes to surface acoustic wave velocity and an increase in defects to variations in build laser power, then identified changes to the component microstructure caused by variations in build laser scan strategy. This was carried out using the detected probe light intensity and the measured surface acoustic velocity.
\end{abstract}

\section{Introduction}

As additive manufacturing (AM) processes develop and start to be used in more high-value industries, such as biomedical, automotive and aerospace, the need to inspect and understand the material properties of the manufactured components will continue to grow $^{[1,2]}$. Selective laser melting (SLM) is an AM process capable of manufacturing metallic components with high precision and fine microstructure ${ }^{[3]}$.

The relationship between the microstructure and the mechanical properties of superalloys capable of performing at high temperatures is well understood ${ }^{[4]}$. The microstructure of AM components, however, varies significantly to those manufactured using more traditional methods such as casting. This is due to the high and variable thermal gradients that occur during the build process of each layer. This variable microstructure, combined with the propensity of the current class of build systems to manufacture parts with high levels of structural defects, can lead to parts with unpredictable and often inferior mechanical performance. For this reason, new research is required that is aimed at detecting and controlling the microstructure of AM components ${ }^{[3]}$.

The layer-by-layer nature of AM processes provides a unique opportunity for an intermediate layer inspection to take place. Several of these inspections could be combined to obtain a volumetric dataset and thereby produce a digital twin of the component being manufactured. The primary requirement for such an inspection would be that it is both non-contact and non-destructive. A range of ultrasonic, optical and thermal nondestructive testing (NDT) techniques have been considered for such an intermediate layer inspection in the past ${ }^{[5-7]}$. It was demonstrated that they were capable of detecting simple structural defects, such as cracks and pores, but failed to classify them and provide any information on the component microstructure. From this, there is a distinct need for an NDT technique that is capable of probing the material properties of the component and providing the AM machine user with some understanding of the microstructure of what is being built.

Spatially resolved acoustic spectroscopy (SRAS) is a laser ultrasonic technique that uses surface acoustic waves (SAWs) to probe the elastic properties of the component. In addition, it generates a separate optical map of the surface being inspected. Previously, SRAS has been used to measure the grain orientation of large-grained materials such as nickel, titanium and silicon ${ }^{[8-10]}$. As an NDT technique, SRAS is uniquely placed to address many of the challenges instigated by SLM. It has the capability to inform upon the microstructural texture of the part ${ }^{[11]}$, the ability to differentiate between surface and subsurface defects ${ }^{[12]}$ and the potential to measure on rough surfaces ${ }^{[13]}$. Hirsch et al conducted a preliminary study into the feasibility of localised reworking of recognised defects, based on SRAS data ${ }^{[14]}$. The technique and instrumentation is described in greater depth in a previous study ${ }^{[15]}$. This article focuses on how SRAS can be used to detect and classify a wide range of defects found within SLM components, such as cracks, pores and how it can provide an insight into component microstructure.

\section{Instrumentation}

The primary elements of the SRAS system described above are: a pulsed generation laser (Q-switched Nd:YAG at $1064 \mathrm{~nm}$, repetition rate of $2 \mathrm{KHz}$, pulse energy of $7.5 \mu \mathrm{J}$ at the sample); a mask used to define the acoustic wavelength; a detection laser (continuous wave at $532 \mathrm{~nm}, 60 \mathrm{~mW}$ at the sample); and a knife edge detector (KED). The generation laser in conjunction with the mask rapidly heats the sample within its thermoelastic regime. This generates a SAW of wavelength, $\lambda$, that is defined by the fringe spacing imaged onto the sample surface. The frequency, $f$, of the SAW travelling

\footnotetext{
- Based on a paper presented at NDT 2018, the 57th Annual British Conference on NDT, Nottingham, UK, September 2018.

This paper was winner of the William Gardner Award.
}

Don Pieris, Rikesh Patel, Paul Dryburgh, Matthias Hirsch, Wenqi Li, Steve D Sharples, Richard J Smith and Matt Clark are with Optics and Photonics, Faculty of Engineering, University of Nottingham, Nottingham, UK.

Don Pieris, Rikesh Patel, Paul Dryburgh, Matthias Hirsch and Adam $T$ Clare are with the Advanced Component Engineering Laboratory (ACEL), Faculty of Engineering, University of Nottingham, Nottingham, UK. 
across the sample is measured by monitoring the perturbation of the detection laser via the KED. Having set the acoustic wavelength and measured the corresponding frequency, the SAW velocity, $v$, is then calculated using Equation (1). This calculated velocity is primarily dependant on the elasticity of the area under excitation, thus in crystalline materials this allows the structure of the grains to be imaged. A more in-depth description of how the system operates can be found in the previously published literature ${ }^{[15-17]}$ :

$$
v=f \lambda \text {. }
$$

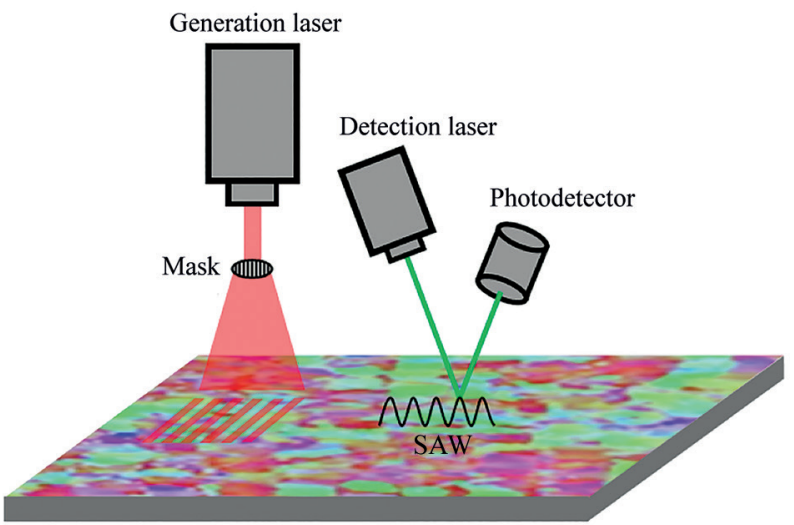

Figure 1. Schematic outlining the $1064 \mathrm{~nm}$ generation laser, the mask used to define the acoustic wavelength, the $532 \mathrm{~nm}$ detection laser and the photodetector. The acoustic wavelength of the current SRAS system is set to $24 \mu \mathrm{m}$. The acoustic generation patch is $200 \mu \mathrm{m}$ in diameter and the optical detection spot is $8 \mu \mathrm{m}$ in diameter

\section{Methodology}

The following sections cover the investigation into the capability of the SRAS instrument to detect changes to a component within an SLM build context. The investigation was carried out using two experiments designed to vary the SLM build parameters.

The first experiment varied the laser power incident on the samples during manufacture. The SRAS system was then used to identify the effect of this change. These samples were also used to investigate the capability of the system to detect surface and subsurface defects.

The second experiment varied the build laser scan strategy while keeping all other build parameters constant. The SRAS instrument was then used to identify these changes on the surface of the samples. Furthermore, the data was used to measure and characterise the defects found on the surface of the samples.

\section{SAW velocity and defect density variation study}

\subsection{Sample preparation}

Smith et $a l^{[12]}$ manufactured eleven samples with the aim of detecting changes to the SAW velocity caused by variations in build laser power. The samples were manufactured using a Renishaw AM250 SLM machine, which had a laser spot size of $70 \mu \mathrm{m}$ and used a Ti-6Al-4V powder feedstock. All of the samples were $10 \mathrm{~mm} \times 10 \mathrm{~mm} \times 10 \mathrm{~mm}$ cubes manufactured with alayer thickness of $30 \mu \mathrm{m}$, a hatch spacing of $75 \mu \mathrm{m}$ and a scan speed of $600 \mathrm{~mm} / \mathrm{s}$.
The build laser power was varied across the samples, from $70 \mathrm{~W}$ to $200 \mathrm{~W}$. The samples were then polished to a mirror finish and inspected using SRAS.

\subsection{Results}

This section presents the results obtained from the specimens outlined earlier using the SRAS instrument. The detection of changes to build parameters was discussed and correlated to changes in either the SRAS optical return or velocity measurement. Following that, defects within the component were detected.

Due to the method by which the SRAS data was gathered, a typical SRAS scan provides two distinct datasets. The optical return is outlined on the left in Figure 2 and the velocity map is shown on the right.
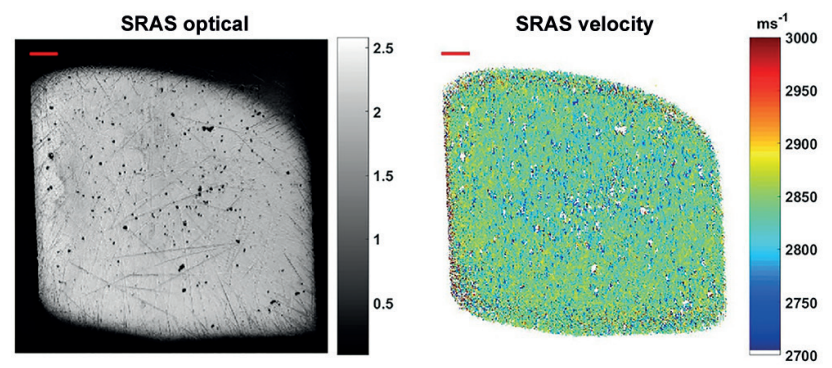

Figure 2. Example SRAS dataset from sample number 8 ( $140 \mathrm{~W})$. Optical return showing several surface-breaking defects (left) and velocity return of SRAS (right). The optical dataset is a voltage measurement based on the light intensity returned to the KED. Defects can be discerned from the dataset but, critically, the microstructural texture development can also be observed ( $1 \mathrm{~mm}$ scale bar)

\subsubsection{Variations in surface defect density and SAW velocity}

The top row of Figure 3 illustrates how the SRAS optical return provided indications of surface defects, such as scratches, cracks and pores. The velocity maps on the bottom row of Figure 3 illustrate the richer stream of acoustic data, which gives some indication of texture change across the samples manufactured with varying build powers. The mean velocity was calculated for each sample inspected using SRAS and was presented in Figure 4. It was clear that as the incident laser power increased across each build, the mean velocity also increased. This increase, however, was not linear and stabilised at the higher energy inputs. It is worth noting
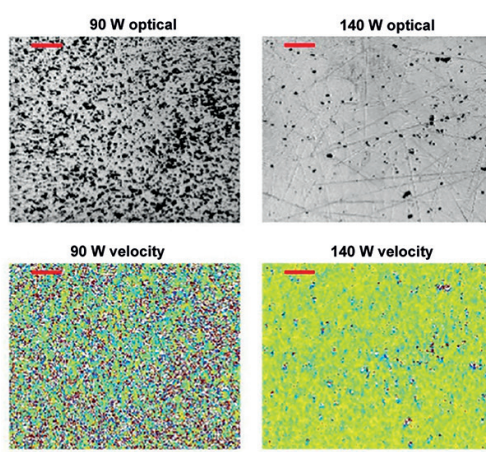

$140 \mathrm{~W}$ velocity

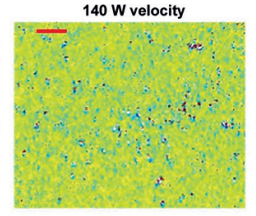
190 W optical

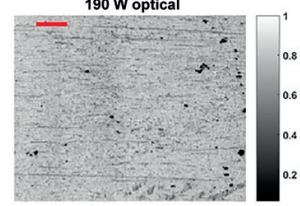

190 Welocity

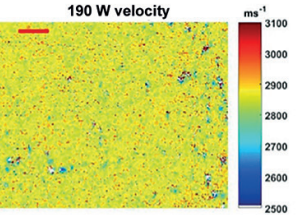

Figure 3. Optical return providing an indication of surface defects but not providing any information regarding material properties (top) and velocity return showing variations across the range of samples (bottom) $(0.5 \mathrm{~mm}$ scale bar) 


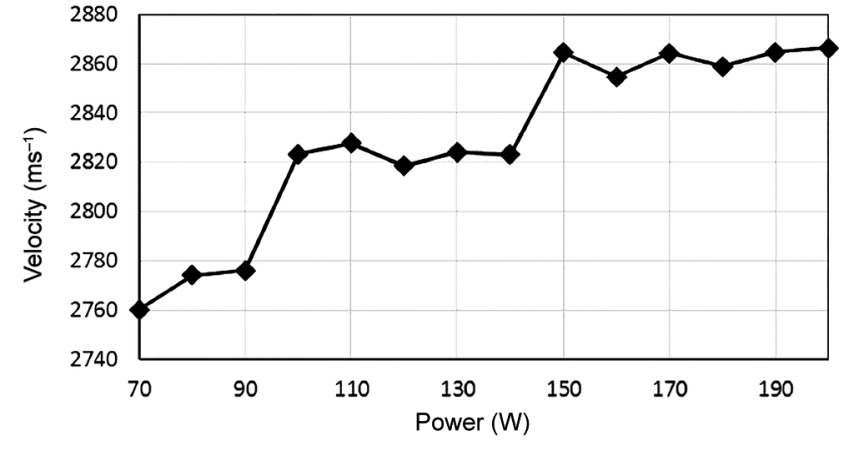

Figure 4. Relationship between input laser power and mean SAW velocity

that, although not considered in this section, this could be due to a reduction in porosity or, more interestingly, due to the development of a preferential grain orientation.

\subsubsection{Surface and subsurface pore detection}

Figure 5(a) illustrates the optical SRAS image of the $190 \mathrm{~W}$ sample. A small section of this sample is more closely inspected in Figure 5(c). The optical defects observed here are confirmed using the scanning

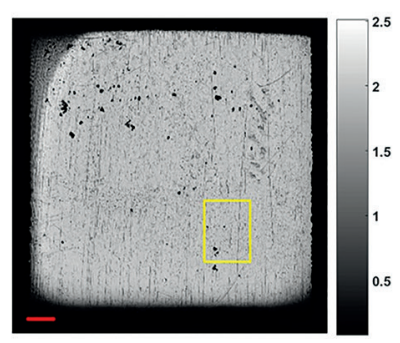

(a)

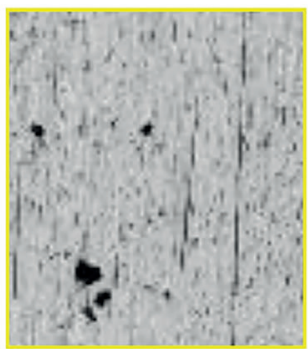

(c)

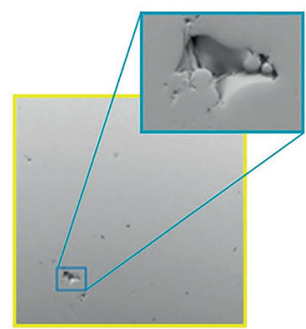

(e)

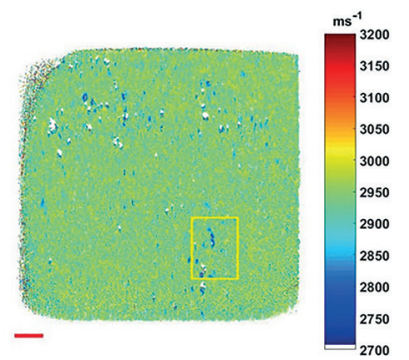

(b)

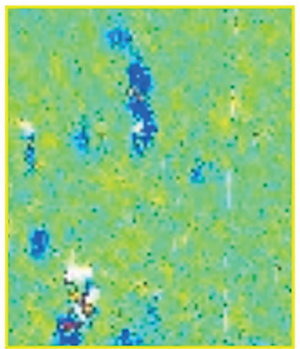

(d)

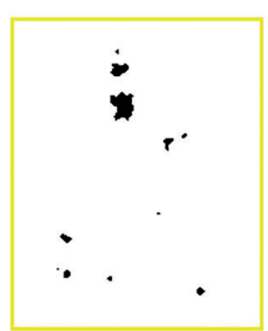

(f)
Figure 5. $190 \mathrm{~W}$ sample: (a) optical return (1 $\mathrm{mm}$ scale bar); (b) velocity map (1 $\mathrm{mm}$ scale bar); (c) close-up optical view of surface defects; (d) close-up of the velocity map of surface and potential subsurface defects; (e) SEM image of surface defect and a zoomed in view of the defect (inset); and (f) X-ray computed tomography (XCT) image of confirmed subsurface defects at approximately $60 \mu \mathrm{m}$ depth electron microscopy (SEM) image in Figure 5(e). The velocity map of the sample is shown in Figure 5(b) and a closer view is shown in Figure 5(d). Several acoustic dropouts can be observed in the upper half of Figure 5(d), which are not observed in the optical images of Figures 5(a) and 5(c). The X-ray computed tomography (XCT) image in Figure 5(f) shows the region just below that in Figures 5(c) and 5(d). The darker area in the XCT image correlates well with the top half of the velocity images, Figures 5(b) and 5(d), but does not correspond with the SRAS optical and SEM images, confirming that the acoustic drop was due to a subsurface feature.

\subsection{Discussion}

Varying the laser power directly varied the energy incident on the fabricated sample. Based on the SRAS results presented in Section 4.2.1, it was clear that the variation of laser power, and thereby energy input, caused changes within the component that are observed using the measured SAW velocity. In this case, the laser power also caused changes to the surface defect density, which are clearly observed in the SRAS optical return.

The combination of the SRAS optical and the velocity results in Section 4.2.2 provided an indication that some subsurface defects can be observed. The velocity map (left) in Figure 6 illustrates the SAW velocity data. The Lamb wave dispersion curve (bottom right) for a $24 \mu \mathrm{m}$ wavelength in titanium illustrates the normal velocity range for titanium in the right-hand square, where $d>\lambda$. With respect to the cross-section (top right) and the left-hand square illustrated in the dispersion curve, it is clear that in the thin platelike region above the pore the SAW wave transformed into a Lamb wave, specifically the fundamental anti-symmetric mode $\mathrm{a}_{0}$. This, in conjunction with the XCT data presented in Figure 5(f), confirms that these are indeed subsurface defects.

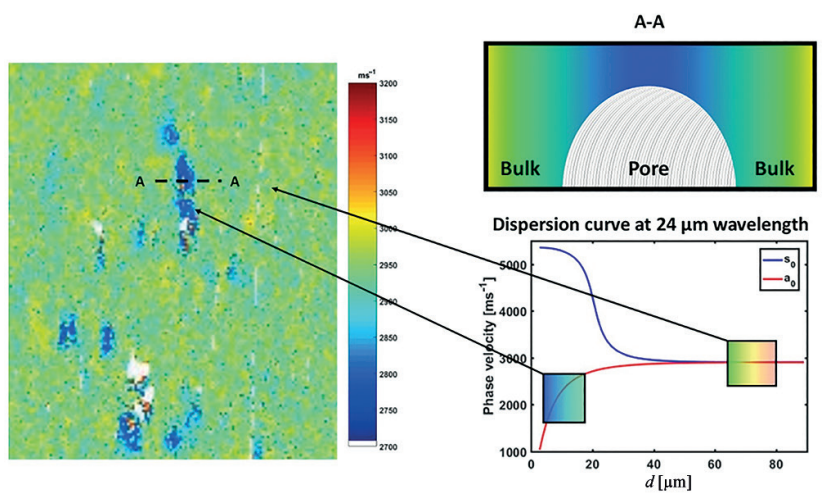

Figure 6. SRAS velocity map of the region with a subsurface pore (left) alongside a theoretical cross-section and dispersion curve for a $\mathbf{2 4} \mu \mathrm{m}$ wavelength in titanium (right)

\section{Microstructure variation study}

\subsection{Sample preparation}

Hirsch et al ${ }^{[11]}$ manufactured a second set of samples, this time with the aim of detecting changes to the microstructure caused by the variations in hatch pattern used by the build laser. In this case, a ReaLizer SLM 50 was used with the nickel superalloy CM247LC feedstock. The laser power was set to $100 \mathrm{~W}$ at a speed of $400 \mathrm{~mm} / \mathrm{s}$, with a hatch spacing of $50 \mu \mathrm{m}$ and a laser spot size of $50 \mu \mathrm{m}$. Each build layer consisted of four equally sized square islands. The angle of the scan lines within these islands was varied across the four samples manufactured. The hatch angles used were $00^{\circ}, 15^{\circ}, 30^{\circ}$ and $45^{\circ}$. Figure 7 shows a schematic drawing of the $00^{\circ}$ and $45^{\circ}$ samples. 
$00^{\circ}$ hatch rotation

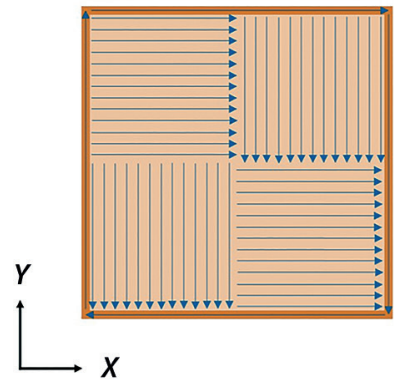

$45^{\circ}$ hatch rotation

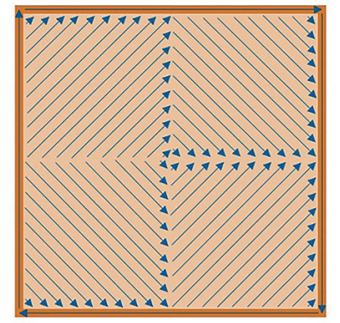

Figure 7. Schematic plan view of: (a) $00^{\circ}$; and (b) $45^{\circ}$ hatch angle samples with equally sized square islands, illustrating how the scan lines are consistently aligned to the set angle across each layer

\subsection{Results}

This section presents the effect that changes to the hatch pattern had on the SRAS results obtained. This data was processed further to classify the defects found on the surface of the sample. Finally, changes to the component microstructure are discussed with relation to the SRAS velocity plots and island boundaries defined during the building of the samples.

\subsubsection{Defect size and density variation}

Figure 8 indicates how the SRAS optical return (top row) can be used to clearly identify surface defects such as cracks and pores. The velocity maps (bottom row) illustrate the wide range of velocity variations observed using SRAS. It is clear that changes to the hatch angle during manufacture can be detected using SRAS optical images as variations in the crack/pore density. The velocity maps provide a more information-rich dataset, illustrating different regions of the island scan strategies as clusters of varying velocities.

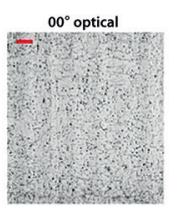

$00^{\circ}$ velocity

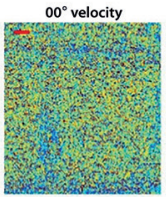

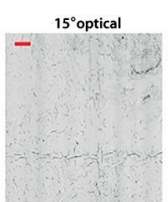

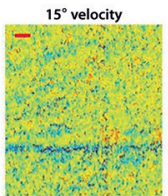

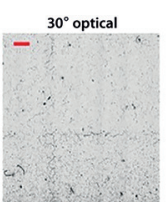

$30^{\circ}$ velocity

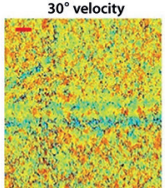

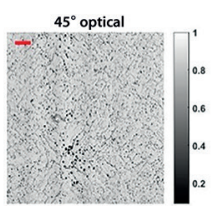

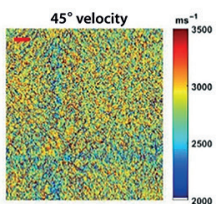

Figure 8. Optical images of SRAS scans of varying hatch angle samples showing some variations in porosity (top row) and velocity maps of varying hatch angle samples showing clear indications of island boundaries as regions of low velocity (bottom row) $(0.5 \mathrm{~mm}$ scale bar)
Using a combination of the SRAS velocity and the optical data, a binary dataset of the defects observed on the $00^{\circ}$ hatch angle sample was obtained. Following some image processing of this dataset, parameters such as the pore location, area, diameter, width and perimeter were extracted.

The calculated defect aspect ratio was used to distinguish between cracks and pores, where cracks would have a higher aspect ratio. Circularity was used to classify pores, where a gas pore would have relatively high circularity. Similarly, the defect count (Figure 9 (right)) could be used as an acceptance criterion, where components with a given number of defects above a certain threshold would not be accepted.
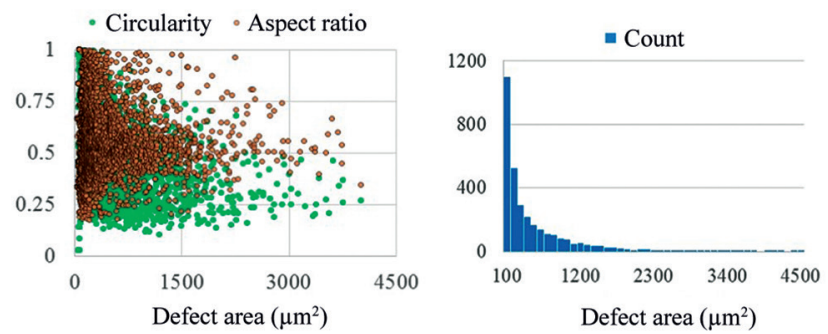

Figure 9. Defect data used to calculate the normalised circularity and aspect ratio (left) and the number of defects found as ranked by their area (right)

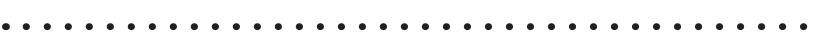

Once several categories of defects were observed, based on the information presented in Table 1, the cause of defects could be identified and action could be taken to prevent them in subsequent layers. Armed with information such as the location, size and shape of the defects, a repair strategy could be implemented in a manner similar to that discussed by Hirsch et al ${ }^{[14]}$.

As a comparison to the SRAS optical data, optical microscopy images were obtained. In Figure 10, the SRAS optical dataset suggests higher defect rates than those suggested by traditional optical microscopy. This is primarily due to the sensitivity of the laser-based measurement, which not only makes the SRAS optical dataset more sensitive to subtle features but also makes it

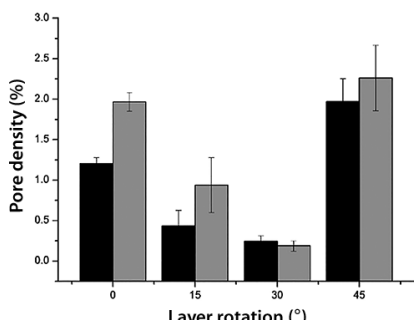

(a)

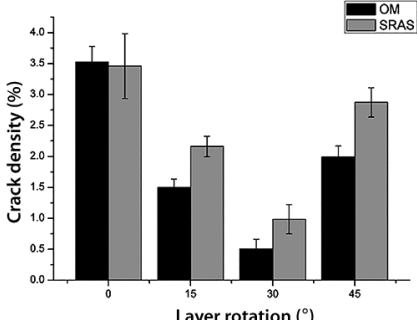

(b)
Figure 10. Comparison of surface cracks and pore density between SRAS and optical microscopy

Table 1. Primary defects in selective laser melting adapted from Everton et al ${ }^{[18]}$

\begin{tabular}{|c|c|c|c|}
\hline Metal powder bed defect & Description & Typical sizes & Reference \\
\hline Spherical pores & $\begin{array}{c}\text { Entrapped gas pores within the bulk of the material. } \\
\text { Material dependent }\end{array}$ & Minimum 5-20 $\mu \mathrm{m}$ & {$[19,20]$} \\
\hline Acicular pores & Pores in between layers of the AM process & $50-500 \mu \mathrm{m}$ & {$[19,20]$} \\
\hline Unfused powder & The melt pool varies in size and unfused powder is present & Satellite powder clumps: $100-150 \mu \mathrm{m}$ & [21] \\
\hline Cracking & $\begin{array}{c}\text { Cracks can be within the component or a disconnection } \\
\text { of the part from the baseplate is seen }\end{array}$ & $\begin{array}{l}\text { Parts on bed: residual stress in the range } \\
\text { of materials yield strength }\end{array}$ & {$[22,23]$} \\
\hline
\end{tabular}


more sensitive to normal variations in background reflectivity. Distinguishing between these will be an important step in the industrialisation of SRAS.

The results presented show that the SRAS instrument was not only capable of detecting typical mechanical defects, such as cracks and pores, but that it was also sensitive to the small changes in the component microstructure. This was seen from the square island boundaries observed in the velocity plots of Figure 8 as lower velocity regions.

\subsubsection{Microstructure variation}

Carter et al ${ }^{[26]}$ utilised electron backscatter diffraction to assess the predominate crystalline orientations in similarly processed CM247LC. The central regions of the hatching islands were seen to have elongated grains with a strong $\left\{\begin{array}{lll}0 & 0 & 1\end{array}\right\}$ orientation. The island boundaries had a finer grain and more chaotic texture with $\left\{\begin{array}{lll}1 & 1 & 0\end{array}\right\}$ and $\left\{\begin{array}{lll}1 & 1 & 1\end{array}\right\}$ orientations dominating. Li et al $l^{[17]}$ have previously reported on the relationship between material elastic properties, crystalline orientation and SAW velocity. Using the same forward model to predict the acoustic slowness surfaces from elastic constants allows the mean velocity to be plotted as a function of crystalline orientation, as shown in Figure 11. Comparing this trend of higher velocities having an orientation closer to $\left\{\begin{array}{lll}0 & 0 & 1\end{array}\right\}$ to the velocity plots given in Figure 8 suggested good agreement with the findings of Carter et al[26]
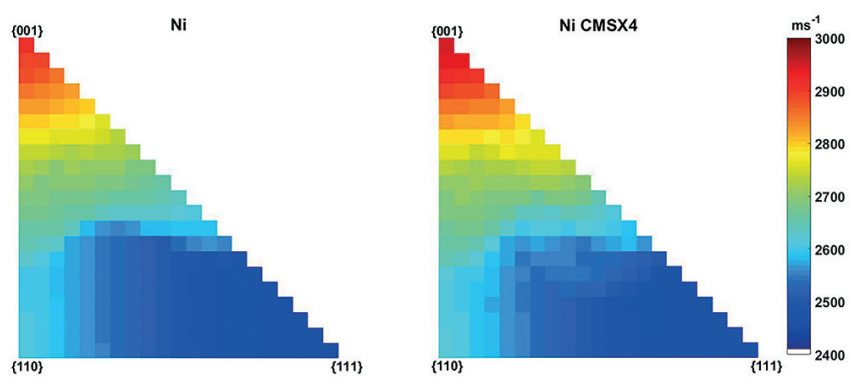

Figure 11. Average velocity maps of all plane families of nickel (left) and nickel superalloy CMSX4 (right), which were calculated based on the elastic constants determined by Salama and Alers ${ }^{[24]}$ for pure nickel and Zhang et $a^{[25]}$ for CMSX4

In addition to the small changes detected across the island boundaries, one highly significant anomaly was detected. A small uncharacteristic line of low velocity was observed inside the north west island of the $30^{\circ}$ sample (Figure 12). Upon closer inspection of the build taking place frameby-frame, it was observed how the scan laser overshot its assigned island on a layer. It was confirmed that this was what was observed in the SRAS velocity data of the $30^{\circ}$ sample. The effect of such build anomalies cannot be detected using an optical technique, emphasising the need for a microstructure sensitive NDT tool.

The authors acknowledge that the 'elephant in the room' is the applicably of the technique to optically rough surfaces. Continuing development of the speckle knife edge detector (SKED) [27] promises to herald a practical solution for carrying out a SRAS inspection on an optically rough surface, by measuring the differences in light intensity across a detector array to unravel the speckle pattern. Some recent work by Patel et al ${ }^{[28]}$ illustrated how a SRAS system using a SKED can not only inspect as-built SLM surfaces but also how the system can easily be integrated into an SLM build chamber.

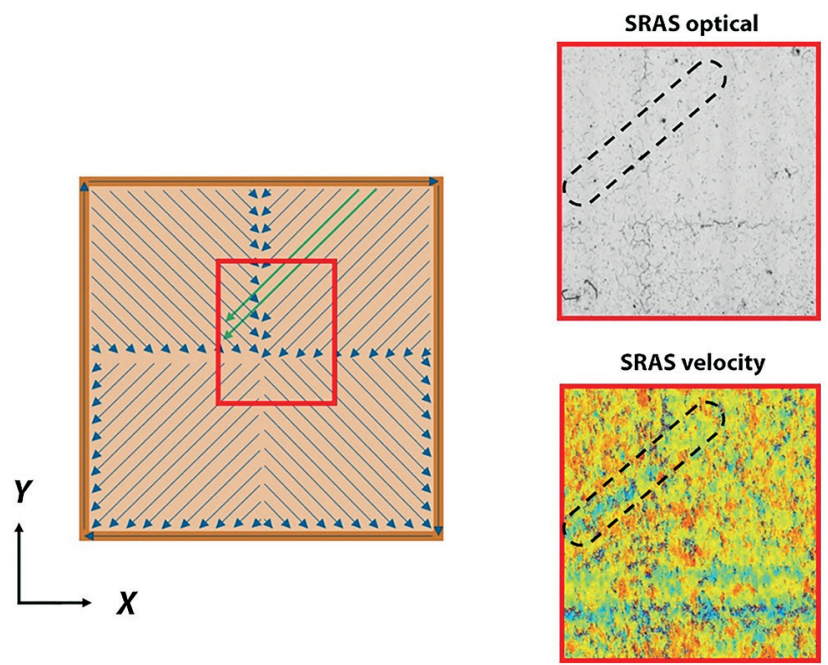

Figure 12. Plan view of the overall scan strategy for a $30^{\circ}$ sample. The region in which the scan lines overshoot the assigned island is highlighted with green arrows alongside both SRAS optical and velocity maps of the same region, which are highlighted in red. Outlined in black, the optical image shows no indication of the scan lines, whereas the velocity map clearly illustrates a change in this region

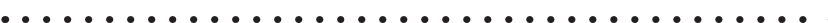

\section{Conclusions}

Based on the results discussed, it is clear that a SRAS instrument is capable of detecting both changes to laser power and hatching strategy in AM components. It is shown that the changes to the build laser power can have an impact on the average surface wave velocity of the component. The SRAS instrument is capable of detecting subsurface defects and some changes to the component microstructure as a change in SAW velocity.

Finally, it is also shown that a combination of SRAS optical and velocity data can be used to count, size and classify defects commonly found in AM parts. Combined with the capability to detect changes to the microstructure, this enables the instrument to be used as part of a feedback loop that is capable of varying build parameters on the fly to minimise defects and optimise parameters. This paves the way for a new generation of SLM AM machines with integrated online inspection platforms such as SRAS.

\section{References}

1. L C Zhang, D Klemm, J Eckert, Y L Hao and T B Sercombe, 'Manufacture by selective laser melting and mechanical behaviour of a biomedical Ti-24Nb-4Zr-8Sn alloy', Scripta Materialia, Vol 65, No 1, pp 21-24, 2011.

2. K N Amato et al, 'Microstructures and mechanical behaviour of Inconel 718 fabricated by selective laser melting', Acta Materialia, Vol 60, No 5, pp 2229-2239, 2012.

3. L Thijs, K Kempen, J P Kruth and J van Humbeeck, 'Finestructured aluminium products with controllable texture by selective laser melting of pre-alloyed AlSi10Mg powder', Acta Materialia, Vol 61, No 5, pp 1809-1819, 2013.

4. I A Essienubong, O Ikechukwu, P O Ebunilo and E Ikpe, 'Material selection for high-pressure (HP) turbine blade of conventional turbojet engines', American Journal of Mechanical and Industrial Engineering, Vol 1, No 1, pp 1-9, 2016.

5. H Rieder, M Spies, J Bamberg and B Henkel, 'On- and offline ultrasonic inspection of additively manufactured components', Proceedings of the 19th World Conference on Non-Destructive 
Testing (WCNDT 2016), Munich, Germany, 13-17 June 2016.

6. M Islam, T Purtonen, H Piili, A Salminen and O Nyrhilä, 'Temperature profile and imaging analysis of laser additive manufacturing of stainless steel', Physics Procedia, Vol 41, pp 835-842, 2013.

7. H Krauss, T Zeugner and M F Zaeh, 'Layerwise monitoring of the selective laser melting process by thermography', Physics Procedia, Vol 56, pp 64-71, 2014.

8. R Smith, S Sharples, W Li, M Clark and M Somekh, 'Orientation imaging using spatially resolved acoustic spectroscopy', Journal of Physics: Conference Series, Vol 353, p 012003, 2012.

9. W Li et al, 'Orientation characterisation of aerospace materials by spatially resolved acoustic spectroscopy', Journal of Physics: Conference Series, Vol 520, p 012017, 2014.

10. R Patel, W Li, R J Smith, S D Sharples and M Clark, 'Orientation imaging of macro-sized polysilicon grains on wafers using spatially resolved acoustic spectroscopy', Scripta Materialia, Vol 140, pp 67-70, 2017.

11. M Hirsch et al, 'Meso-scale defect evaluation of selective laser melting using spatially resolved acoustic spectroscopy', Proceedings of the Royal Society A: Mathematical, Physical and Engineering Sciences, Vol 473, No 2205, p 20170194, 2017.

12. R J Smith, M Hirsch, R Patel, W Li, A T Clare and S D Sharples, 'Spatially resolved acoustic spectroscopy for selective laser melting', Journal of Materials Processing Technology, Vol 236, pp 93-102, 2016.

13. S O Achamfuo-Yeboah, R A Light and S D Sharples, 'Optical detection of ultrasound from optically rough surfaces using a custom CMOS sensor', Journal of Physics: Conference Series, Vol 581, p 012009, 2015.

14. M Hirsch et al, 'Targeted rework strategies for powder bed additive manufacture', Additive Manufacturing, Vol 19, pp 127$133,2018$.

15. R J Smith, W Li, J Coulson, M Clark, M G Somekh and S D Sharples, 'Spatially resolved acoustic spectroscopy for rapid imaging of material microstructure and grain orientation', Measurement Science and Technology, Vol 25, No 5, p 055902, 2014.

16. S D Sharples, M Clark and M G Somekh, 'Spatially resolved acoustic spectroscopy for fast non-contact imaging of material microstructure', Optics Express, Vol 14, No 22, pp 10435-10440, 2006.

17. W Li, S D Sharples, R J Smith, M Clark and M G Somekh, 'Determination of crystallographic orientation of large grain metals with surface acoustic waves', The Journal of the Acoustical Society of America, Vol 132, No 2, pp 738-745, 2012.

18. S K Everton, M Hirsch, P Stravroulakis, R K Leach and A T Clare, 'Review of in-situ process monitoring and in-situ metrology for metal additive manufacturing, Materials and Design, Vol 95, pp 431-445, 2016.

19. S Tammas-Williams, H Zhao, F Léonard, F Derguti, I Todd and P B Prangnell, 'XCT analysis of the influence of melt strategies on defect population in Ti-6Al-4V components manufactured by selective electron beam melting, Materials Characterization, Vol 102, pp 47-61, 2015.

20. L Thijs, F Verhaeghe, T Craeghs, J van Humbeeck and J P Kruth, 'A study of the microstructural evolution during selective laser melting of Ti-6Al-4V', Acta Materialia, Vol 58, No 9, pp 3303$3312,2010$.

21. H Niu and I T Chang, 'Instability of scan tracks of selective laser sintering of high-speed steel powder', Scripta Materialia, Vol 41, No 11, pp 1229-1234, 1999.
22. P Mercelis and J-P Kruth, 'Residual stresses in selective laser sintering and selective laser melting', Rapid Prototyping Journal, Vol 12, No 5, pp 254-265, 2006.

23. M F Zaeh and G Branner, 'Investigations on residual stresses and deformations in selective laser melting', Production Engineering, Vol 4, No 1, pp 35-45, 2010.

24. A Salama, G A Alers, 'Third-order elastic modulus of pure nickel and some dilute copper-nickel alloys', IEEE Transactions on Sonics and Ultrasonics, p 28, 1969.

25. X Zhang, P R Stoddart, J D Comins and A G Every, 'Hightemperature elastic properties of a nickel-based superalloy studied by surface Brillouin scattering, Journal of Physics: Condensed Matter, Vol 13, No 10, pp 2281-2294, 2001.

26. L N Carter, C Martin, P J Withers and M M Attallah, 'The influence of the laser scan strategy on grain structure and cracking behaviour in SLM powder-bed fabricated nickel superalloy', Journal of Alloys and Compounds, Vol 615, pp 338347, 2014.

27. S D Sharples, R A Light, S O Achamfuo-Yeboah, M Clark and M G Somekh, 'The SKED: speckle knife edge detector', Journal of Physics: Conference Series, Vol 520, p 012004, 2014.

28. R Patel et al, 'Imaging material texture of as-deposited selective laser-melted parts using spatially resolved acoustic spectroscopy', Applied Sciences, Vol 8, No 10, p 1991, 2018. 